sches, zeitbeanspruchendes Vorgehen. Die ohnehin erheblich vorgeschädigten Kinder werden so einer starken Operationsbelastung ausgesetzt. Die intraoperative Sichtbarmachung der extrahepatischen Gallenwege durch Injektion einer Methylenblaulösung in die Gallenblase ist imstande, eine schnelle Information der jeweils vorliegenden anatomischen Gegebenheiten zu vermitteln und auf diese Weise die Operationszeit und damit das Operationsrisiko zu vermindern.

Anhand von 2 Fällen wird die Untersuchungsmethode demonstriert.

Fall 1. 5 Monate altes Kind. Verdacht auf kongenitale Gallengangsatresie. Nach intraoperativer Injektion von Methylenblaulösung in die Gallenblase prompte Darstellung der normal angelegten, enggestellten extrahepatischen Gallenwege. Der Befund wird durch ein intraoperatives Cholangiogramm bestätigt. Probeexcision aus der Leber: Riesenzellhepatitis.

Fall 2. 51/2 Monate altes Kind. Klinisch: Gallengangsatresie. Die intraoperative Farbdarstellung ergibt eine typische Atresie des Ductus hepaticus mit Stop $1 \frac{1}{2} \mathrm{~cm}$ proximal der Cysticuseinmündung. Zudem ergibt sich eine Blauanfärbung des rechten Leberlappens aufgrund direkt in die Gallenblase eintretender Gallengänge. Therapie: Hepato-Jejunostomie (Y-Anastomose).

\title{
11 c. Die Versorgung der Schenkelhalsfraktur mit der Femurschaftendoprothese
}

\author{
B. Friedriorr und R. Zwirner (a.G.) \\ Chirurgische Klinik der Universität Würzburg \\ (Direktor: Prof. Dr. E. Kern)
}

Mit allen bekannten Osteosyntheseverfahren bei der medialen Schenkelhalsfraktur kann man bestenfalls eine Übungsstabilität erreichen. Für den alten Menschen liegt auch darin noch eine ernste Gefahr; denn er kann meist nur dann mobilisiert werden, wenn er seine Extremitäten belasten darf. Daher sollte heute die Versorgung der medialen Schenkelhalsfraktur bei diesen Patienten mit der Femurschaftendoprothese angestrebt werden.

Vorteile der Femurschaftendoprothese bei der Versorgung der medialen Schenkelhalsfraktur:

1. Kurzer und schonender Eingriff.

2. Kein Blutverlust, also kein Blutersatz nötig.

3. Sofortige Belastbarkeit nach der Operation.

4. Kurzer Krankenhausaufenthalt.

Das Instrumentarium und die Operationstechnik werden durch entsprechendes Bildmaterial aufgezeigt. 